\title{
PRODUCTION OF FAST NEUTRON WITH A PLASMA FOCUS DEVICE
}

\section{Moshe Gai *}

Laboratory for Nuclear Science at Avery Point, University of Connecticut, 1084 Shennecossett Rd, Groton, CT 06340-6097.

and

Department of Physics, WNSL Rm 102, Yale University, PO Box 208124,

272 Whitney Avenue, New Haven, CT 06520-8124.

http://astro.uconn.edu, E-mail: moshe.gai@yale.edu

Before its demise DIANA Hi-TECH, LLC, demonstrated the use of two 50 kJoule Plasma Focus devices for the copius production of fast neutrons, x-rays and radio-isotopes. Such a device is suitable for fast neutron non invasive interogation of contra-band materials including hidden nuclear materials. It could be particularly useful for a fast and fail safe interogation of large cargo containers, or in merchant marine port of entries. The performance and fast neutron production ( 2.5 or $14 \mathrm{MeV}$ at $10^{11}$ or $10^{13}$ per pulse, respectively) of the two PF50 Plasma Focus devices produced by DIANA HiTECH, LLC, are discussed.

International Workshop on Fast Neutron Detectors and Applications

April, 3 - 6, 2006

University of Cape Town, South Africa

${ }^{*}$ Work Supported by USDOE Grant No. DE-FG02-94ER40870. 


\section{Introduction}

The Plasma Focus (PF) phenomenon was independently discovered by J. Mather [1] and N. V.Filippov [2] in the late 50's. Since then advances were made [3] in many laboratories that studied the capabilities of PF for producing short pulses (10 - $100 \mathrm{~ns})$ of X-rays, neutrons and fast ions depending on PF mode of operation. The production of radio-isotopes in PF has recently received attention $[4,5]$. Commercial PF devices are now available from All-Russia Research Institute of Automatics [6]. PF operation can be briefly described as a five stage process (see Fig. 1) with the appearance of high-energy ions and nuclear reactions occurring in the last stage. In Fig. 2 we show a picture of the PF25 operated at DIANA HiTECH, LLC, in North Bergen, New Jersey,.

These studies [7] suggest the following empirical formula for the flux of ions with ( $\mathrm{E}>0.1$ $\mathrm{MeV}): d \Phi / d E \approx E^{-m}(2<m<3)$. This spectral dependence of ions trapped in the plasma leads to the observed neutron spectrum emitted from a plasma focus device operating with deuterium gas. The data provide evidence that the yield of fast beams as well as the reaction yields in plasma $\left(Y_{p}\right)$ and/or with external targets $\left(Y_{t}\right)$ placed inside a PF, all scale with the square of the energy $(\mathrm{W})$ stored in the capacitor bank [8].

The experimental group at Steven Tech has achieved high efficiency (yield per kJ of stored energy) for the production of short-lived radioisotopes in a plasma environment [9] using a Mathertype PF device with a capacitor bank energy of $\mathrm{W}=7 \mathrm{~kJ}(18,000$ Volts $)$ and a gas mixture pressure of $\mathrm{p} \approx 5$ Torr. Gas mixtures were composed of low-Z (LZ) isotopes (hydrogen or deuterium) mixed with high-Z (HZ) isotopes. The obtained results are shown in Fig. 3, from which it is clear that a broad range of radioisotopes, with large yield, can be produced in a PF device. A relatively small PF-machine, operated at a discharge power of $\mathrm{W}=7 \mathrm{~kJ}$, produced $10^{6}$ to $5 \times 10^{8}$ radio-nuclei per pulse, while with a medium size machine, operated at discharge power of, $\mathrm{W}=70 \mathrm{~kJ}$, one can expect a hundred times higher yields.

\section{The Stevens Tech and DIANA's PF5, PF25 and PF50}

\section{Production of Fast Neutrons}

Of particular interest are PF devices operating with a deuterium gas or deuterium plus tritium gas mixture. They yield 2.5 and $14 \mathrm{MeV}$ fast neutrons, respectively. Since the cross section for $\mathrm{t}(\mathrm{d}, \mathrm{n}) \alpha$ reaction is 100 times larger than $\mathrm{d}(\mathrm{d}, \mathrm{n})$ reaction, the production rate of $14 \mathrm{MeV}$ neutrons is 100 times larger under the same PF conditions. The pulse repetition rate of of PF is mainly dictated by the charging time of the RC capacitors, and the time duration of the pulse can be manipulated to a small extent by changing the geometry of the plasma electrodes $[10,11,12]$. It must be emphasized that the neutron yield of the PF device is due to the instabilities produced in the last stage of the plasma (stage 5 in Fig. 1) which causes pulse to pulse fluctuation of the absolute neutron yield and the spatial location of the neutron source. Hence applications of the PF must include monitoring these effects.

The rates of neutrons produced in two PF50 devices operating with deuterium gas were measured at DIANA HiTECH, LLC, by measuring the activation of silver by the moderated neutrons to produce ${ }^{108} \mathrm{Ag}(\tau=2.41 \mathrm{~min})$ and ${ }^{110} \mathrm{Ag}(\tau=24.6 \mathrm{sec})$. The activation method was calibrated 


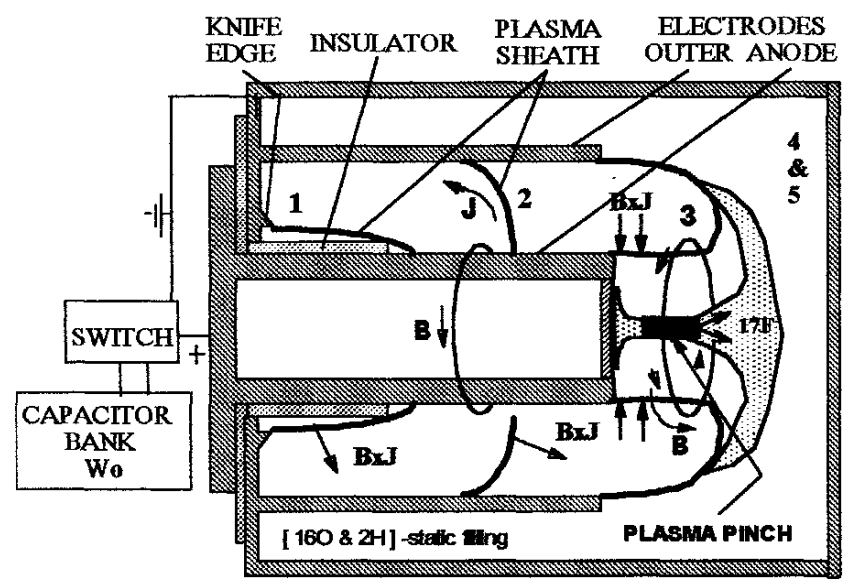

Figure 1: Schematic operation of a plasma focus header (used for the production of ${ }^{17} F$ ). We indicate the sequence of plasma sheath positions. The central electrode diameter is approximately $50 \mathrm{~mm}$, the chamber is filled with oxygen plus deuterium gas mixture at $\mathrm{p}=0.1-7$ Torr. The plasma develops in stages: 1 the plasma sheath is formed, 2 - the plasma sheath moves toward the anode nozzle ( $v \approx 10^{5} \mathrm{~m} / \mathrm{s}$ ), 3 - the sheath arrives at the end of the anode and rearranges into a cylinder with a conical opening, 4 - the plasma is compressed at the axis $\left(10^{23}\right.$ ions $\left./ \mathrm{m}^{3}\right), 5$ - the plasma column quickly develops instabilities associated with high energy acceleration (with large nuclear reactivity and intense X-ray emission). Small (20 - 300 microns) plasma domains are created. The domains have above solid state densities, and temperature of $\mathrm{kT}$ $>3 \mathrm{keV}$ and magnetic fields sufficient to trap ions with kinetic energy of up to $5 \mathrm{MeV} /$ nucleon.

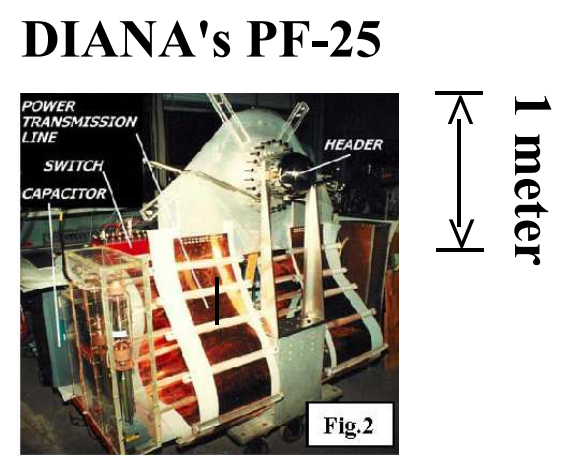

Figure 2: A picture of the PF25 operated at DIANA, HiTECH, LLC.

using an intense Pu-Be source at the Wright Laboratory at Yale University. The obtained neutron production rate with the two PF50 device tested at DIANA HiTECH, LLC, confirmed (after scaling) the production rate predicted for the PF70 device $\left(10^{11} \mathrm{n} /\right.$ pulse for $\mathrm{d}$ - $\mathrm{d}$ fusion and $10^{13} \mathrm{n} / \mathrm{pulse}$ for d-t fusion) as shown in Fig. 3. The two PF50 devices were operated over a long period (a few days) at a rate of $1 \mathrm{~Hz}$. The control system of the PF was shielded and contained in a Faraday cage due to the intense associated electromagnetic pulse.

\section{References}

[1] Mather, J.W, Physics of Fluids 8,336-341 (1965).

[2] Filippov, N.V., Filippova, T.L, and Vinogradov, V.P., Nuclear Fusion Suppl. "Dense and High 


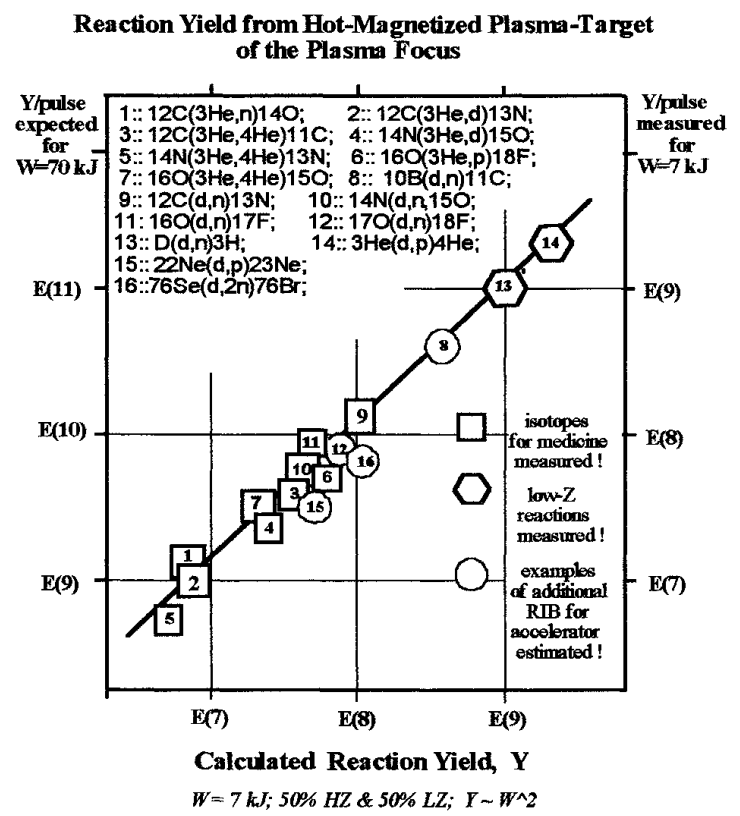

Figure 3: Measured production rates (with PF7) and predicted yield (for PF70). Neutrons (2.5 MeV) are produced in $\mathrm{PF}$ loaded with deuterium gas (13). For a mixture of $\mathrm{d}+\mathrm{t}$ the production rate of $14 \mathrm{Mev}$ neutrons is 100 times larger.

Temperature Plasma in Non-Cilindrical Z-Pinch" in 1st Int. Conf. on Plasma Fusion Physics, Salzburg-1962, Publ. by IAEA as Nuclear Fusion Suppl, 1962, vol.2, pp577-587.

[3] See for example Proc. 2nd IAEA Research Coordination Meeting, Dense Magnetized Plasma, Nukleonika, Vol. 51, 2006.

[4] J.S. Brzosko, K. Melzacki, C. Powell, M. Gai, R.H. France III, J.E. McDonald, G.D. Alton, F.E. Bertrand, and J.R. Beene. "Breeding $10^{10}$ Radioactive Nuclei in Compact Plasma Focus Device; A.I.P. CP576(2001)277.

[5] E.Angeli, A. Tartari, M. Frignani, V. Molinari, D. Mostacci, F. Rocchi, and M. Summi, "Production of Radioisotopes Within a Plasma Focus Device", Nucl. Tech. Rad. Protec. 20, 1(2005)33.

[6] All-Russia Research Institute of Automatics (VNIIA); http://www.vniia.ru/eng/index.html.

[7] Nardi V., Bortolotti A., Brzosko J.S., Esper M, Luo, CM, Pedrielli F., Powdl C, and Zeng D., IEEE Trans. On Plasma Science 16,368-373 (1988).

[8] Brzosko, J.S., Degnan, J.,Filippov, N.V., Freeman, B.L., Kiuttu, J., Mather, J.W., "Comments on the Feasibility of Achieving Scientific Break-Even with a Plasma Focus Machine", Current Trends in International Fusion Research edited by R. Panarella, Plenum Press, New York, 1997, pp. 11-32.

[9] Brzosko IS., and Nardi V., Physics Lett. A155, 162-168, (1991), Physics Lett A192, 250-257 (1994), Phys. Plasmas 2, 1259-1269 (1995).

[10] Sing Lee and Adrian Serban; IEEE Trans Plasma Sci 24(1996)1101.

[11] J.S. Brzosko, B.V. Robouch, L. Ingrosso, A. Bortolotti, and V. NBardi; Nucl. Inst. Meth. B72(1992)119.

[12] J.S. Brzosko, J.R. Brzosko, B. Robouch, L. Ingrosso; Phys. Plasma 2(1995)1259. 